\title{
Lazarevicite-type short-range ordering in ternary III-V nanowires
}

\author{
M. Schnedler, ${ }^{1}$ I. Lefebvre, ${ }^{2}$ T. Xu, ${ }^{2,3}$ V. Portz, ${ }^{1}$ G. Patriarche, ${ }^{4}$ J.-P. Nys, ${ }^{2}$ S. R. Plissard, ${ }^{2,5}$ P. Caroff, ${ }^{2,6}$ M. Berthe, ${ }^{2}$ H. Eisele, ${ }^{7}$ \\ R. E. Dunin-Borkowski, ${ }^{1}{ }^{1}$ Ph. Ebert, ${ }^{1}$ and B. Grandidier ${ }^{2}$ \\ ${ }^{1}$ Peter Grünberg Institut, Forschungszentrum Jülich GmbH, 52425 Jülich, Germany \\ ${ }^{2}$ Département ISEN, Institut d'Electronique, de Microélectronique et de Nanotechnologie (IEMN), CNRS, \\ UMR 8520, 41 Boulevard Vauban, 59046 Lille Cedex, France \\ ${ }^{3}$ Key Laboratory of Advanced Display and System Application, Shanghai University, \\ 149 Yanchang Road, Shanghai 200072, People's Republic of China \\ ${ }^{4}$ Laboratoire de Photonique et de Nanostructures (LPN), CNRS, Université Paris-Saclay, route de Nozay, 91460 Marcoussis, France \\ ${ }^{5}$ Laboratoire d'Analyse et d'Architecture des Systèmes (LAAS), CNRS, Université de Toulouse, \\ 7 Avenue du Colonel Roche, 31400 Toulouse, France \\ ${ }^{6}$ Department of Electronic Materials Engineering, Research School of Physics and Engineering, The Australian National University, \\ Canberra, Australian Capital Territory 0200, Australia \\ ${ }^{7}$ Institut für Festkörperphysik, Technische Universität Berlin, Hardenbergstrasse 36, 10623 Berlin \\ (Received 20 June 2016; revised manuscript received 9 October 2016; published 14 November 2016)
}

\begin{abstract}
Stabilizing ordering instead of randomness in alloy semiconductor materials is a powerful means to change their physical properties. We used scanning tunneling and transmission electron microscopies to reveal the existence of an unrecognized ordering in ternary III-V materials. The lazarevicite short-range order, found in the shell of $\operatorname{InAs}_{1-x} \mathrm{Sb}_{x}$ nanowires, is driven by the strong $\mathrm{Sb}$-Sb repulsion along $\langle 110\rangle$ atomic chains during their incorporation on unreconstructed $\{110\}$ sidewalls. Its spontaneous formation under group-III-rich conditions of growth offers the prospect to broaden the limited classes of ordered structures occurring in III-V semiconductor alloys.
\end{abstract}

DOI: 10.1103/PhysRevB.94.195306

Binary III-V compound semiconductors cover only discrete values of materials properties, such as band gaps and lattice parameters. Many technological applications require, however, materials with properties intermediate between those of the binary compounds. Commonly this is achieved by creating solid solutions of different binary compounds. The electronic properties of such ternary or quaternary III-V alloys depend, however, sensitively on the chemical ordering. Although alloys with long-range order may exhibit significantly deviating band gaps from random alloys with identical compositions [1,2], alloys with short-range order (SRO) have been shown to affect the carrier localization in the alloys and modify their optical properties [3]. Studying the nature of SRO in III-V alloys and the physical mechanisms leading to its spontaneous formation is thus of prime importance to further understand the interplay between atomic-scale structures and electronic properties in these alloys.

Chemical ordering has been investigated rather well for thin films of III-V compound semiconductors [2,4,5]. Recently, ordering was found to occur in III-V semiconductor nanowires (NWs) too [6,7]. However, despite the considerable interest in a better control of the ordering and the large number of possible ordered spatial arrangements that are predicted by the space-group theory, only a few ordered structures have been reproducibly stabilized from the parent zinc-blende (ZB) lattice: $\mathrm{CuPt}$, triple period, $\mathrm{CuAu}-\mathrm{I}$, chalcopyrite, and famatinite [8]. This restriction is intimately related to the limited numbers of achievable surface reconstructions and the limited ranges of the growth parameters, such as temperature, growth rate, III/V ratio, and substrate orientation. Recent developments in the epitaxial growth of III-V semiconductors, such as the ones achieved with liquid-droplet epitaxy or obtained during the growth of semiconductor nanowires, offer the potential to reach unattained growth conditions and growth regimes $[9,10]$. This situation raises the question whether new ordered structures could be tailored in III-V semiconductor materials.

To address this question, we used scanning tunneling microscopy (STM) and scanning transmission electron microscopy (STEM) to provide direct evidence of SRO in ZB $\mathrm{InAs}_{1-x} \mathrm{Sb}_{x}$ nanowires. In addition to a CuPt SRO typically found in $\mathrm{InAs}_{1-x} \mathrm{Sb}_{x}$ thin films [11], we identify a lazarevicitetype [12] SRO. This SRO prevails due to the shell growth on $1 \times 1$ unreconstructed sidewalls. It arises from the repulsive $\mathrm{Sb}-\mathrm{Sb}$ interaction that prevents an incoming $\mathrm{Sb}$ atom from binding at a $\langle 110\rangle$ atomic row with a $\mathrm{Sb}$ atom as the nearest neighbor. As (110) surfaces are encountered in III-V semiconductor thin films, the lazarevicite-type SRO should not be limited to nanowires, thereby extending the groups of ordered structures occurring in ternary III-V materials.

$[\overline{1} \overline{1} \overline{1}]$-oriented $\mathrm{ZB} \operatorname{InAs}_{1-x} \mathrm{Sb}_{x} \mathrm{NW}$ segments were grown by $\mathrm{Au}$ droplet-assisted gas source molecular beam epitaxy (MBE) on top of wurtzite (WZ) InAs NWs, which were first nucleated on InP stems on $\operatorname{InP}(111) B$ substrates [13]. The growth temperature was set at $410{ }^{\circ} \mathrm{C}$. During the growth, the antimony fraction was controlled by a valve opening, measured by a flux gauge, and the Sb composition $x$ in the $\operatorname{InAs}_{1-x} \mathrm{Sb}_{x}$ NW segments was precisely evaluated by x-ray energydispersive spectroscopy (XEDS) point-scan analysis after the growth. For the NWs of interest in this paper, $x$ ranged between 0.1 and 0.2 . Figure 1(a) illustrates the overall NW structure schematically for $x=0.1$. The bottom WZ InAs segment has a sixfold symmetry and consists of $m$-plane $\{10 \overline{1} 0\}$ sidewall facets separated by small $\{11 \overline{2} 0\}$ facets. The top $\operatorname{InAs}_{1-x} \mathrm{Sb}_{x}$ NW segment crystallizes in the ZB structure and shows six equivalent nonpolar $\{110\}$ sidewall surfaces. In this segment, 
(a)
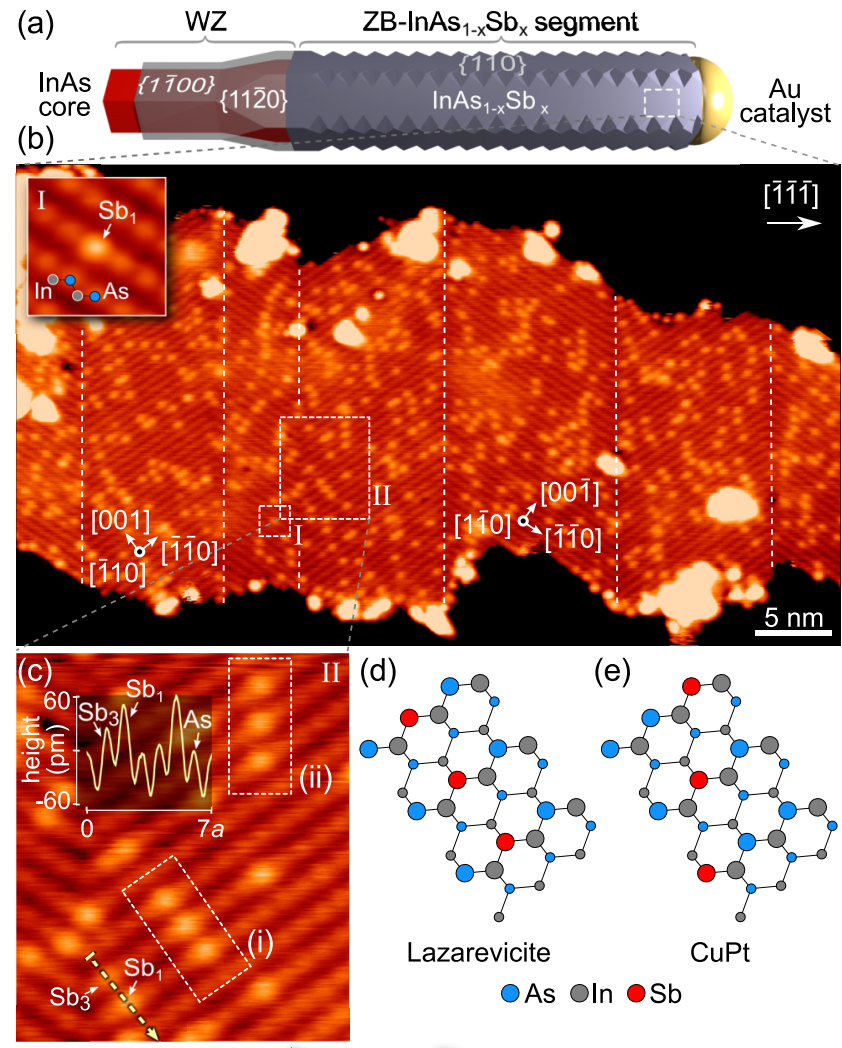

(e) Lazarevicite OAs Oln OSb

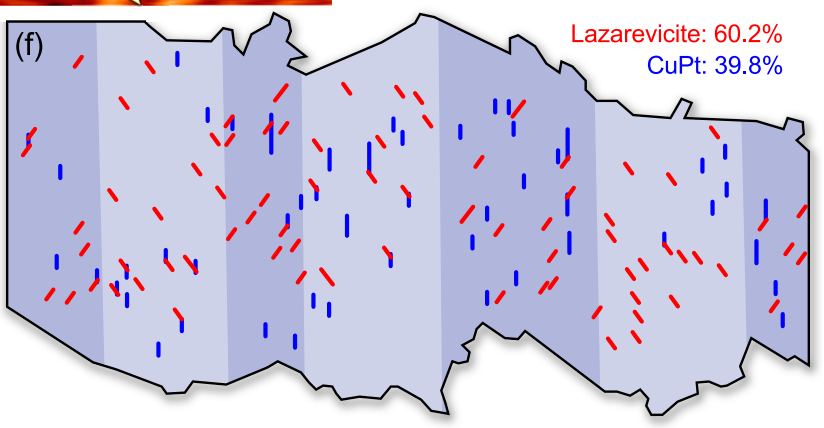

FIG. 1. (a) Schematic of a $[\overline{1} \overline{1} \overline{1}]$ grown $\operatorname{InAs}_{0.90} \mathrm{Sb}_{0.10} / \mathrm{InAs}$ nanowire. (b) Atomically resolved constant-current STM image of the sidewall surface measured at $77 \mathrm{~K}(-3 \mathrm{~V}$ sample voltage and $10 \mathrm{pA}$ tunnel current). The image shows the filled dangling bond states above the surface anions. The bright atomically localized contrast features arise from $\mathrm{Sb}$ atoms incorporated on anion sites. Twin boundaries are marked by dashed vertical lines. The inset [marked I in (b)]: high-resolution STM image $(-2 \mathrm{~V}, 700 \mathrm{pA})$ of one $\mathrm{Sb}_{\mathrm{As}}$ atom in the surface layer. The location of the atomic zigzag chain of alternating anion and cations is indicated by a ball model. (c) High-resolution STM image $(-2 \mathrm{~V}, 700 \mathrm{pA})$ of area II in (b). Sb atoms in the first layer $\left(\mathrm{Sb}_{1}\right)$ and third layer $\left(\mathrm{Sb}_{3}\right)$ are visible. Local lazarevicite- [12] and CuPt-type ordered areas are labeled (i) and (ii), respectively. (d) and (e) illustrate the respective atomic models. (f) Spatial distribution of CuPt (blue) and lazarecivite (red) SROs.

a sawtooth faceting occurs at the edges between the neighboring $\{110\}$ sidewalls. This sawtooth faceting is connected with pseudoperiodic twin boundaries and consists of either alternating $\{111\}$ and $\{\overline{1} \overline{1} \overline{1}\}$ or $\{001\}$ and $\{00 \overline{1}\}$ surfaces.

After the NW growth, the samples were capped at room temperature with a thin layer of arsenic for protection against oxidation, transferred through air into an UHV system equipped with STM, and decapped there. Then the NWs were cleaved off against a freshly cleaned $\operatorname{Si}(111) 7 \times 7$ surface [14]. As shown previously, this decapping procedure does not change the $\mathrm{Sb}$ concentration profile and morphology of the NWs [13].

Figure 1(b) shows an atomically resolved STM image of the sidewall facet of an $\operatorname{InAs}_{0.90} \mathrm{Sb}_{0.10} \mathrm{NW}$ segment, whose bulk composition was determined by $\mathrm{x}$-ray energy-dispersive spectroscopy point analysis. The sidewall facet with a surface composition of $\operatorname{InAs}_{0.86} \mathrm{Sb}_{0.14}$ exhibits twinned $\mathrm{ZB}$ domains 5-10 $\mathrm{nm}$ wide, separated by twin boundaries (dashed vertical lines), consistent with the structure illustrated in Fig. 1(a). In each domain, parallel atomic rows are visible, which are the hallmark of $\{110\}$ surfaces. Although a row consists of alternating anions and cations, as depicted in the overlying schematic ball model of the inset of Fig. 1(b), only the occupied dangling bonds above the surface anions (As and $\mathrm{Sb}$ atoms) are imaged at negative sample bias [15].

Notably, some anion atomic positions appear brighter in the STM images. They are the signature of Sb atoms on anion lattice sites. Sb atoms at and below $\{110\}$ surfaces of GaAs (and InAs) are known to exhibit an intense bright contrast in STM images due to relaxation and electronic effects [16]. Most of the Sb atoms induce a height change of $60 \mathrm{pm}$ [see the $\mathrm{Sb}_{1}$ peak in height profile in Fig. 1(c)], suggesting that the majority of $\mathrm{Sb}$ atoms are located in the first surface layer $\left(\mathrm{Sb}_{1}\right.$ concentration of $\left.14 \%\right)$. $\mathrm{Sb}$ on the second (subsurface) plane is expected to give rise to two equally shifted surface anions but with a smaller height change [17,18]. Such contrast features are, however, not observed. Instead, individual weaker maxima occur, which are centered exactly on a surface anion position and have a height of $\sim 30$ pm only [see height profile in Fig. 1(c)] . Their symmetry and intensity are compatible with $\mathrm{Sb}$ on the third (subsurface) plane [labeled $\mathrm{Sb}_{3}$ in Fig. 1(c)] [17,19].

The atomically resolved view of the individual chemical species enables a chemical mapping and hence the investigation of ordering in a ternary NW. Using this chemical map we derived the two-dimensional pair correlation function (PCF) for Sb atoms [Fig. 2(a)]. The PCF exhibits large values above 1 along the [001] and [001] directions, indicating the frequent occurrence of $\mathrm{Sb}$ pairs across the atomic chains. In contrast, nearest- and second-nearest-neighbor $\mathrm{Sb}$ pairs within the atomic chains in the $\pm[110]$ directions occur rarely, as indicated by values significantly smaller than 1 . Hence, the ternary $\operatorname{InAs}_{0.90} \mathrm{Sb}_{0.10}$ alloy of the NW is not statistically distributed but rather exhibits a SRO, defined by an ordering or correlation vector $\left(\underline{v}_{\mathrm{O}}\right)$ in the [001] direction and an anticorrelation vector $\left(\underline{v}_{\mathrm{AO}}\right)$ in the [110] direction. The PCF allows for estimating the ordered domain size in the [001] direction on the basis of the Gaussian FWHM to be $3.4 a$.

In order to determine where the formation of the SRO takes place, STEM images were acquired for NWs with different $\mathrm{Sb}$ contents. For the $\mathrm{InAs}_{0.9} \mathrm{Sb}_{0.1} \mathrm{NWs}$ characterized with STM, the typical reflections of the (110) zone axis of the ZB structure were observed in the fast Fourier transform (FFT) images, but no superstructure diffraction spots appeared in the NW bulk, in contrast to the SRO found with STM on the sidewall surface. In contrast, for $\operatorname{InAs}_{1-x} \mathrm{Sb}_{x} \mathrm{NWs}$ with higher 


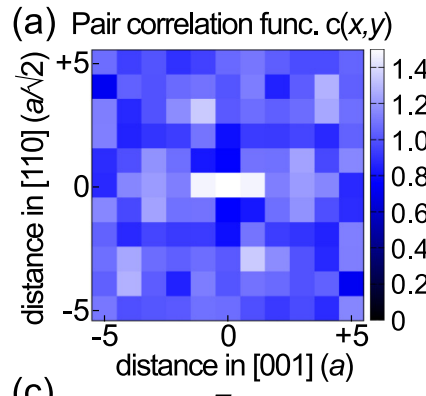

(c)

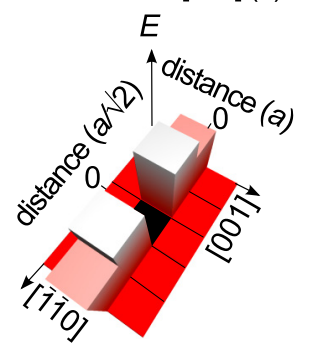

(e)

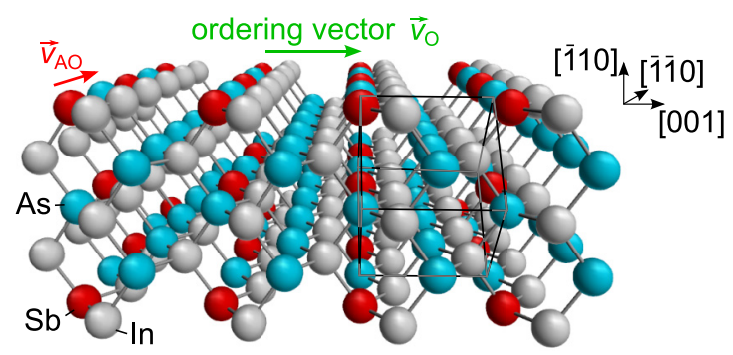

FIG. 2. (a) Two-dimensional pair correlation function $c(x, y)$ 's of $\mathrm{Sb}$ atoms in $\mathrm{InAs}_{0.90} \mathrm{Sb}_{0.10} \mathrm{NWs}$ measured on the $\{110\}$ sidewall facet. Values above (below) 1 indicate a higher (lower) than statistically expected occurrence of $\mathrm{Sb}$ pairs (scale on the right). (b) Mean force potential $W(r)=-k T \ln [c(x, y)]$ derived from the experimentally measured PCF. (c) Calculated two-dimensional pair interaction energy of $\mathrm{Sb}$ pairs. (d) Comparison of the experimental (open symbols, right scale) and calculated (filled symbols, left scale) pair interaction energies along the [110] direction. (e) Lazarevicite crystal structure with indications of the ordering $\left(\underline{v}_{\mathrm{O}}=[001]\right)$ and anticorrelation $\left(\underline{v}_{\mathrm{AO}}=[110]\right)$ vectors on a $\{110\}$ plane. The cubic unit cell is indicated on the right side.

Sb composition $x$ between 0.15 and 0.20 , the FFT of the STEM image in Fig. 3(a) shows superstructure spots (red circles) in addition to the expected reflections of the (110) zone axis of the ZB structure labeled in black [Fig. 3(b)]. They correspond to [001] and [110] orderings, similar to what is observed in the STM images. These ordering peaks were not observed near the Au catalyst droplet, although the Sb composition was similar [Fig. 3(c)]. Therefore, we conclude that the ordering occurs in the laterally grown NW shell.

Although no superstructure spots were found in the FFT images of the lower bulk Sb composition ( $\operatorname{InAs}_{0.9} \mathrm{Sb}_{0.1} \mathrm{NWs}$ ), the STM images of the sidewall surface clearly demonstrate the presence of ordering. This apparent discrepancy can be resolved as follows. First, the lateral overgrowth is smaller, and hence, the shell thinner for lower Sb concentrations. This is corroborated by the NW morphology: At low Sb concentrations $\left(\operatorname{InAs}_{0.90} \mathrm{Sb}_{0.10}\right)$ the NW morphology is dominated by a sawtooth faceting, whereas at high $\mathrm{Sb}$ concentrations

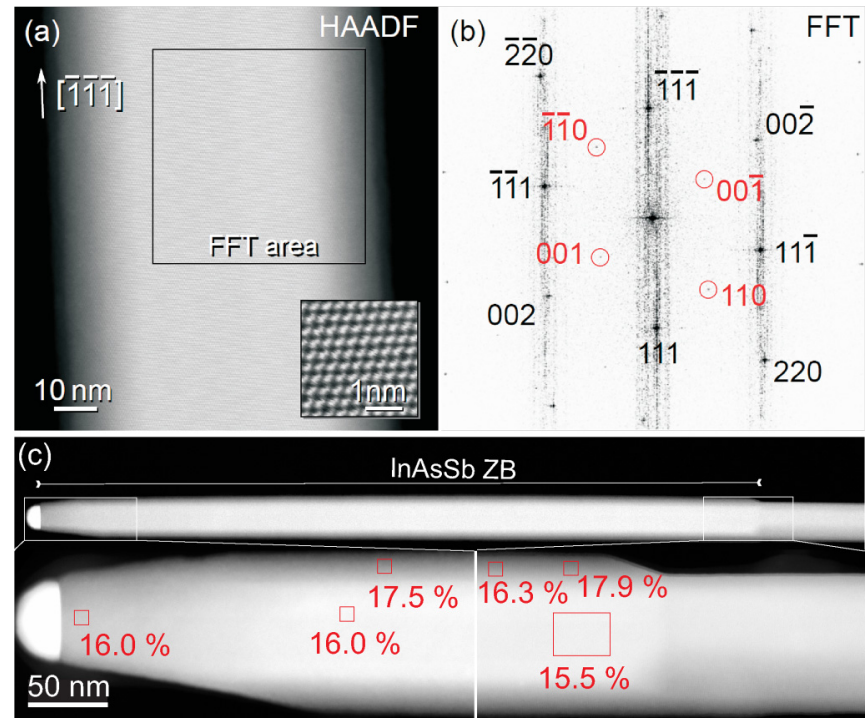

FIG. 3. (a) Atomically-resolved scanning transmission electron microscopy image in the high-angle annular dark-field (HAADF) mode of an $\operatorname{InAs}_{0.84} \mathrm{Sb}_{0.16}$ nanowire along a $\langle 110\rangle$ zone axis. The magnified inset shows the dumbbell structure of the atomic columns. The square marks the area used for the FFT shown in (b). Two types of ordering spots occur, labeled $[001] /[00 \overline{1}]$ and $[110] /[\overline{1} \overline{1} 0]$. (c) HAADF-STEM images of a ZB InAs ${ }_{0.84} \mathrm{Sb}_{0.16} \mathrm{NW}$ segment. The concentration of $\mathrm{Sb}$ atoms with respect to the anion concentration was measured by XEDS point scan analyses in the areas marked in red.

( $>0.12)$ straight sidewalls are observed. The sawtooth facets disappear successively during lateral overgrowth [10]. Hence, their persistence at low $\mathrm{Sb}$ compositions (see Fig. 1) indicates a thin shell, which does not show up in the STEM images with sufficient intensity, and thus no superstructure spots can be detected, even if present. Second, the Sb concentration near the sidewall surfaces is higher than at the bulk one $(0.14$ vs 0.10 ). This surface composition of the NW investigated by STM is close to that of the $\mathrm{InAs}_{0.84} \mathrm{Sb}_{0.16} \mathrm{NW}$ [ranging between $15.5 \%$ and $17.9 \%$ as measured by XEDS in Fig. 3(c)] where the superstructure spots were clearly observed in the FFT of the STEM image [Fig. 3(b)]. Therefore, the importance to probe a shell thick enough to unveil the ordering with STEM indicates that the SRO takes place during the lateral overgrowth and precludes its formation at the end of the growth during quenching or during the As-decapping process.

For identifying the type of SRO, we briefly recall the currently known ordering types in ternary ZB III-V semiconductors: The most common one, called layered trigonal or $\mathrm{CuPt}[4,20]$, exhibits on the $\{110\}$ planes either an ordering vector in the $\langle 11 \overline{2}\rangle$ direction or an anticorrelation vector in the [001] direction combined with an ordering vector in the [110] direction. This is not compatible with our structure. Similarly the triple-period structures [2,21] can be ruled out. The chalcopyrite structure [22] has either a $\langle 11 \overline{2}\rangle$ ordering vector or the combined ordering vectors in the $\langle 001\rangle$ and $\langle 1 \overline{1} 0\rangle$ directions, again not describing our case. The famatinite structure [23] has three types of $\{110\}$ planes, but none agrees with our structure. The layered tetragonal or $\mathrm{CuAu}-\mathrm{I}$ structure 
has an ordering vector in $\langle 110\rangle$, also in conflict with our observation.

The SRO is only compatible with a lazarevicite structure, which has on $\{110\}$ planes an ordering vector in the [001] direction and an anticorrelation vector in the [110] direction [12]. A model of the lazarevicite structure is shown in Fig. 2(e) with the (110) surface plane on the top side. It reproduces the features observed in the Sb PCF. In the STM images, this ordering shows up as Sb pairs or alignments along the [001] direction [Fig. 1(d) and the example marked by (i) in the high-resolution STM image in Fig. 1(c)]. In contrast to any III-V semiconductors, the lazarevicite SRO is thus observed here and the question arises why this type of SRO is found in $\operatorname{InAs}_{1-x} \mathrm{Sb}_{x}$ NWs.

In order to identify the physical mechanisms leading to this new type of SRO, we turn to density functional theory calculations using the Vienna ab initio simulation program (version 5.2.11) [24]. For the total energy calculations all electron projector augmented-wave (PAW) pseudopotentials were taken [25] using the Ceperley and Alder approximation [26] parametrized by Perdew and Zunger for exchange and correlation energies (i.e., the Sb, As, In, H1.25, and H.75 PAW potentials) [27]. We used a kinetic-energy cutoff of $312.5 \mathrm{eV}$. The structure of the $(1 \times 1 \times 18)$-ML (where ML represents monolayer) $\langle 110\rangle$-oriented supercell $(\mathrm{H}$ terminated on the backside) was optimized until forces on atoms were smaller than $0.9 \mathrm{meV} / \AA$. The calculated lattice constant of $6.053 \AA$ is in good agreement with the experimental one. Then the total energy of different $\mathrm{Sb}-\mathrm{Sb}$ pair configurations on the InAs(110) surface layer was calculated using a $(3 \times 5 \times 6)$-ML supercell.

Figure 2(c) shows a graphic representation of the energies of different $\mathrm{Sb}-\mathrm{Sb}$ configurations. The lowest-energy $E_{\mathrm{Sb}-\mathrm{Sb},[001]}$ is found for Sb pairs across the atomic chains in the [001] direction. Similar in energy are $\mathrm{Sb}$ pairs incorporated diagonally across the atomic chains $\left(E_{\mathrm{Sb}-\mathrm{Sb},[001]}+1 \mathrm{meV}\right)$. The highest energy occurs for nearest- and second-nearest-neighbor $\mathrm{Sb}$ pairs within the atomic zigzag chains in the [110] direction with an energy of $E_{\mathrm{Sb}-\mathrm{Sb},[001]}+33$ and $E_{\mathrm{Sb}-\mathrm{Sb},[001]}+24 \mathrm{meV}$, respectively. The calculated $\mathrm{Sb}$ pair interaction energy map [Fig. 2(c)] agrees quantitatively with the mean force potential $W(r)=-k T \ln [c(x, y)]$ [28] derived from the experimental PCF $c(x, y)$ [Fig. 2(b)], as visible in Fig. 2(d). This indicates a strong repulsion within the atomic zigzag chains due to the larger size of $\mathrm{Sb}$ atoms as compared with As atoms. Across the atomic chains, the strain can be relaxed better, lowering the pair interaction energy.

The calculated interaction energies are compatible with $\mathrm{Sb}$ pairs along both $\langle 001\rangle$ and $\langle 11 \overline{2}\rangle$ directions. These ordering directions are compatible with the formation of lazareviciteand CuPt-type orderings, respectively [see Figs. 1(d) and 1(e)]. Indeed, locally, $\mathrm{Sb}$ atoms are aligned along the diagonal $\langle 11 \overline{2}\rangle$ direction [example marked (ii) in Fig. 1(c)] corresponding to local insertions of a CuPt SRO, as schematically illustrated in Fig. 1(e) $[29,30]$. Figure 1(f) reveals a lazarevicite-CuPt ordering ratio of about 60:40. Of all $\mathrm{Sb}$ atoms present about $28 \%, 20 \%$, and $52 \%$ exhibit a lazarevicite ordering, a $\mathrm{CuPt}$ ordering, and no ordering, respectively.

In order to understand the preference of the lazarevicite $\mathrm{SRO}$, we turn to the NW growth processes and the incorpora-

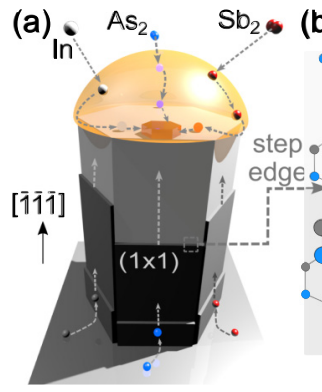

(b)

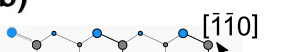

(c)

FIG. 4. (a) Schematic of the growth of $\operatorname{InAs}_{1-x} \mathrm{Sb}_{x}$ nanowires. The shell growth on the $\{110\}$ sidewalls of a $[\overline{1} \overline{1} \overline{1}]$-oriented nanowire occurs through step flow motion of $\langle 112\rangle$-oriented steps. (b) and (c) Atomic models of the $\mathrm{Sb}$ incorporation at the step edge on the sidewall surface. Sb cannot be incorporated in the atomic zigzag chains [position 2 in (b)] if another Sb rests on the neighboring anion lattice site of the same chain (position 1). Hence, the new $\mathrm{Sb}$ atom is deviated into the neighboring chain (e.g., position 3), leading to a [001] ordering vector.

tion mechanisms of the individual atoms. The growth of the NWs by MBE involves not only the direct supply of precursors into the Au droplet, but also impingement (and dissociation) of In atoms and $\mathrm{As}_{2} / \mathrm{Sb}_{2}$ molecules at the sidewalls and the substrate. These adatoms diffuse from the substrate toward the Au droplet [Fig. 4(a)] [31]. A certain fraction is already incorporated on the $\{110\}$ sidewalls of the NW, inducing a lateral overgrowth $[13,32]$. Considering the growth temperature $(683 \mathrm{~K})$, the growth rate $(0.5 \mathrm{ML} / \mathrm{s})$ and the V-III flux ratio of 2 to 3 used here, the lateral overgrowth proceeds in the step flow mode, in analogy to homoepitaxy on GaAs(110) [33]. The islands nucleate preferentially at the bottom of the NWs (leading to a larger diameter there) and grow by step flows in the $[\overline{1} \overline{1} \overline{1}]$ direction [along the NW long axis, Fig. 4(a)]. Under growth conditions on III-V(110) surfaces, the steps are parallel to the $\langle 11 \overline{2}\rangle$ direction [34]. Such steps perpendicular to the NW long axis can also be observed by scanning electron microscopy (SEM) and STM on NW sidewall surfaces [10,13] and are energetically preferred [35]. Hence in the following we have to discuss the incorporation at $\langle 11 \overline{2}\rangle$-oriented steps.

We recall that the surface reconstruction plays a central role in the formation of ordered phases in planar III-V semiconductor growth: For example, III-V(001) $2 \times 3,2 \times 1$, and $2 \times 2$ surfaces result in triple period, $\mathrm{CuPt}_{B}$, and $\mathrm{CuPt}_{A}$ structures, respectively $[2,36-38]$. The $\{110\}$ surfaces of $\mathrm{ZB}$ III-V semiconductors exhibit a relaxed $1 \times 1$ bulklike structure under stoichiometric conditions. Growth of III-V NWs is performed with very low V/III ratios, far off the group-V-rich conditions of planar thin film growth, compatible with a stoichiometric $1 \times 1$ unreconstructed structure. Furthermore, the edge of the $\langle 112\rangle$-oriented steps on the III-V $\{110\}$ cleavage surfaces exhibit a $\times 1$ bulklike structure too [39].

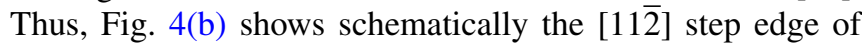
the growing terrace (bottom) on the $1 \times 1\{110\}$ sidewall. We exemplify the situation where one $\mathrm{Sb}$ atom was incorporated already in the previous [112] -oriented atomic row [red atom marked 1 in Fig. 4(b)]. The strong repulsion of Sb-Sb pairs along the atomic zigzag chain in the [110] direction blocks a 
new $\mathrm{Sb}$ atom to be incorporated at lattice position 2 . Hence, the $\mathrm{Sb}$ atom has to sidestep towards one of the neighboring lattice positions (3 or 4 ). If incorporated at position 3 , lazarevicitetype [001] ordering forms [Fig. 4(c)].

The additional occurrence of CuPt SRO can be explained with our growth model too if [001]-oriented step facets (i.e., kinks of $\langle 11 \overline{2}\rangle$ steps) are present or when several Sb atoms are incorporated simultaneously in neighboring atomic chains of kink-free $\langle 11 \overline{2}\rangle$ steps. Interestingly, the material grown on top of CuPt-like ordered $\mathrm{Sb}\langle 11 \overline{2}\rangle$ chains contains no $\mathrm{Sb}$ for the following three to five monolayers in the $[\overline{1} \overline{1} \overline{1}]$ growth direction [Fig. 1(b)]. This emphasizes the strong Sb-Sb repulsion within the atomic chains acting now on several parallel chains simultaneously.

In conclusion, we found a new type of $\mathrm{SRO}$ in $\operatorname{InAs}_{1-x} \mathrm{Sb}_{x}$ NWs that departs from the common CuPt ordering in planar InAs $s_{1-x} \mathrm{Sb}_{x}$ thin films. The ordering is obtained during the growth of the shell as proven for antimony fractions $x$ between 0.14 and 0.18 . The findings provide a possible growth recipe for lazarevicite ordering in thin films by growing on $\{110\}$ substrates miscut in $\langle\overline{111}\rangle$ with low growth fluxes to avoid the simultaneous arrival of two or more $\mathrm{Sb}$ at the $\langle 11 \overline{2}\rangle$ step edges, suppressing the CuPt SRO.

\section{ACKNOWLEDGMENTS}

The authors thank the European Community's Seventh Framework Program (Grant No. PITN-GA-2012316751, "Nanoembrace" Project), the RENATECH network, the Impuls- und Vernetzungsfonds of the HelmholtzGemeinschaft Deutscher Forschungszentren (Grant No. HIRG-0014), the French National Research Agency (TERADOT Project No. ANR-11-JS04-002-01, the Equipex Programs Excelsior ANR-11-EQPX-0015 and Tempos ANR-10EQPX-50), the "Chenguang" Project (Grant No. 13CG42) of the Shanghai Municipal Education Commission and Shanghai Education development Foundation and of the Region NordPas-de-Calais, and the Deutsche Forschungsgemeinschaft (Grant No. SFB 787 TP A4) for financial support, K. A. Dick and X. Wallart for fruitful discussions, and C. Coinon and J.-L. Codron for technical support.
[1] S.-H. Wei and A. Zunger, Phys. Rev. B 39, 3279 (1989).

[2] A. Gomyo, K. Makita, I. Hino, and T. Suzuki, Phys. Rev. Lett. 72, 673 (1994).

[3] J. A. Chan, J. Z. Liu, and A. Zunger, Phys. Rev. B 82, 045112 (2010).

[4] A. Zunger and S. Mahajan, in Handbook on Semiconductors: Materials, Properties and Preparation, edited by S. Mahajan (North-Holland, Amsterdam, 1994), Vol. 3B, pp. 1399-1514.

[5] T. S. Kuan, T. F. Kuech, W. I. Wang, and E. L. Wilkie, Phys. Rev. Lett. 54, 201 (1985).

[6] S. Y. Woo, M. Bugnet, H. P. T. Nguyen, Z. Mi, and G. A. Botton, Nano Lett. 15, 6413 (2015).

[7] D. Ercolani, M. Gemmi, L. Nasi, F. Rossi, M. Pea, A. Li, G. Salviati, F. Beltram, and L. Sorba, Nanotechnology 23, 115606 (2012).

[8] The structure labeled "luzonite" in Ref. [1] is not identical with that of the mineral luzonite $[23,40]$. The correct-type structure denomination is lazarevicite [12]. The minerals luzonite $\mathrm{Cu}_{3} \mathrm{AsS}_{4}$ [41] and famatinite $\mathrm{Cu}_{3} \mathrm{SbS}_{4}$ [42] are the end members of a solid solution series with isotype structures [23]. Since famatinite has been published prior to luzonite, the former name should be used for the type structure.

[9] Z. Y. Zhou, C. X. Zheng, W. X. Tang, J. Tersoff, and D. E. Jesson, Phys. Rev. Lett. 111, 036102 (2013).

[10] J. V. Knutsson, S. Lehmann, M. Hjort, P. Reinke, E. Lundgren, K. A. Dick, R. Timm, and A. Mikkelsen, ACS Appl. Mater. Interfaces 7, 5748 (2015).

[11] H. R. Jen, K. Y. Ma, and G. B. Stringfellow, Appl. Phys. Lett. 54, 1154 (1989).

[12] C. B. Sclar and M. Drovenik, Bull. Geol. Soc. Am. 71, 1970 (1960)

[13] T. Xu, K. A. Dick, S. Plissard, T. H. Nguyen, Y. Makoudi, M. Berthe, J.-P. Nys., X. Wallart, B. Grandidier, and P. Caroff, Nanotechnology 23, 095702 (2012).
[14] T. Xu and B. Grandidier, in Semiconductor Nanowires: Materials, Synthesis, Characterization and Applications, edited by J. Arbiol and Q. Xiong (Woodhead, Sawston, Cambridge, UK, 2015), Vol. 77, pp. 277-304.

[15] R. M. Feenstra, J. A. Stroscio, J. Tersoff, and A. P. Fein, Phys. Rev. Lett. 58, 1192 (1987).

[16] J. Steinshnider, J. Harper, M. Weimer, C.-H. Lin, S. S. Pei, and D. H. Chow, Phys. Rev. Lett. 85, 4562 (2000).

[17] Ph. Ebert, M. Heinrich, M. Simon, C. Domke, K. Urban, C. K. Shih, M. B. Webb, and M. G. Lagally, Phys. Rev. B 53, 4580 (1996).

[18] K.-J. Chao, C.-K. Shih, D. W. Gotthold, and B. G. Streetman, Phys. Rev. Lett. 79, 4822 (1997).

[19] H. A. McKay, R. M. Feenstra, T. Schmidtling, and U. W. Pohl, Appl. Phys. Lett. 78, 82 (2001).

[20] A. Mascarenhas, Spontaneous Ordering in Semiconductor Alloys (Kluwer-Academic-Plenum, New York, 2002).

[21] M. Wu, E. Luna, J. Puustinen, M. Guina, and A. Trampert, Appl. Phys. Lett. 105, 041602 (2014).

[22] S. R. Hall and J. M. Stewart, Acta Crystallogr., Sect. B: Struct. Crystallogr. Cryst. Chem. 29, 579 (1973).

[23] R. V. Gaines, Am. Mineral. 42, 766 (1957).

[24] G. Kresse and J. Furthmüller, Phys. Rev. B 54, 11169 (1996).

[25] P. E. Blöchl, Phys. Rev. B 50, 17953 (1994).

[26] D. M. Ceperley and B. J. Alder, Phys. Rev. Lett. 45, 566 (1980).

[27] T. Xu, M. J. Wei, P. Capiod, A. Díaz Álvarez, X. L. Han, D. Troadec, J. P. Nys, M. Berthe, I. Lefebvre, G. Patriarche, S. R. Plissard, P. Caroff, Ph. Ebert, and B. Grandidier, Appl. Phys. Lett. 107, 112102 (2015).

[28] The mean force potential equals the pair interaction energy in the low-density limit under equilibrium. T. L. Hill, Statistical Mechanics (McGraw-Hill, New York, 1956). 
[29] A. J. Heinrich, M. Wenderoth, K. J. Engel, T. C. G. Reusch, K. Sauthoff, R. G. Ulbrich, E. R. Weber, and K. Uchida, Phys. Rev. B 59, 10296 (1999).

[30] F. Genz, A. Lenz, H. Eisele, L. Ivanova, R. Timm, U. W. Pohl, M. Dähne, D. Franke, and H. Künzel, J. Vac. Sci. Technol., B: Nanotechnol. Microelectron.: Mater., Process., meas., Phenom. 28, C5E1 (2010).

[31] V. G. Dubrovskii, T. Xu, A. Díaz Álvarez, S. R. Plissard, P. Caroff, F. Glas, and B. Grandidier, Nano Lett. 15, 5580 (2015).

[32] M. Hjort, S. Lehmann, J. Knutsson, A. A. Zakharov, Y. A. Du, S. Sakong, R. Timm, G. Nylund, E. Lundgren, P. Kratzer, K. A. Dick, and A. Mikkelsen, ACS Nano 8, 12346 (2014).

[33] P. Tejedor, P. Šmilauer, and B. A. Joyce, Surf. Sci. Lett. 424, L309 (1999).
[34] P. Tejedor, P. Šmilauer, C. Roberts, and B. A. Joyce, Phys. Rev. B 59, 2341 (1999).

[35] J. M. McCoy and J. P. LaFemina, Phys. Rev. B 54, 14511 (1996).

[36] S. Froyen and A. Zunger, Phys. Rev. Lett. 66, 2132 (1991).

[37] A. Gomyo, M. Sumino, I. Hino, and T. Suzuki, Jpn. J. Appl. Phys., Part 2 34, L469 (1995).

[38] S. B. Zhang, S. Froyen, and A. Zunger, Appl. Phys. Lett. 67, 3141 (1995).

[39] M. Heinrich, C. Domke, P. Ebert, and K. Urban, Phys. Rev. B 53, 10894 (1996).

[40] F. Marumo and W. Nowacki, Z. Kristallogr. 124, 1 (1967).

[41] A. Weisbach, Mineralog. Mittheilungen, Jahrbuch k.-k. Geol. Reichsanstalt Wien, 257 (1874).

[42] A. Stelzner, Mineralog. Mittheilungen, Jahrbuch k.-k. Geol. Reichsanstalt Wien, 219 (1873). 